\title{
PREPARING TO TEACH ON THE CLOUD NEXT TIME - ADAPTING TO LESSONS FROM FIRST TIME TEACHING ON THE CLOUD
}

Azad Ali, Indiana University of Pennsylvania, azad.ali@iup.edu

David Smith, Indiana University of Pennsylvania, David.smith@iup.edu

\begin{abstract}
This paper follows up on the discussion of teaching on the cloud that we published in a previous study on the same topic. Our previous paper explained about the issues and challenges that faces the teaching of cloud computing for the first time. Some of these issues are addressed within the framework of technology changes. At the same time, other issues remain outstanding. This paper examines teaching on the cloud from three perspectives technology, organization and environment. It uses a framework called TOE (Technology, Organization and Environment) to explain the factors that influence the teaching of courses on the cloud.
\end{abstract}

Keywords: Cloud Computing, Virtualization, Teaching on the Cloud, Working on the Cloud

\section{INTRODUCTION}

"Cloud computing", "working on the cloud", and "teaching on the cloud" are terms often used to reference accessing computing resources that reside in remote locations which are accessed through broadband or other similar high-speed communications. The word "cloud" here references data centers that have extensive computing resources that are located around the world (Alharthi, et al., 2017, Amburst, et al., 2017).

Cloud computing is often compared with "on premise computing" (Almmajalid, 2017) or "conventional computing" (Amburst, et al., 2010), which refers to computing resources that reside internally on the premises of the organization. In comparison cloud computing offers numerous advantages to the organization, including cost advantages, flexibility, and access of shared resources. Thus, many organizations are moving their computing resources from on premise to the cloud, this is called "moving to the cloud".

However, moving computing resources to the cloud or teaching on the cloud is not without risk and challenges as the steps involved are not always seamless (Gangware \& Ramaswamy, 2015). Even prior to making the move, organizations are often face related issues that they must be addressed (Low \& Chen, 2011). Given this, there are recommendations to consider factors related to the technology involved, the organization, and the environment of cloud computing (Harris \& Khan, 2018) prior to moving to the cloud.

This paper illustrates the experience of a faculty in preparing to teach on the cloud for a second time. A number of issues and challenges were previously reported by the faculty's first time teaching on the cloud (Ali \& Smith, 2018). Two factors encouraged the faculty to research about this topic: first, the speed of changes that accompany the development of cloud computing. And second, the enormous volume of new teaching materials that can now be found for cloud computing.

The paper addresses the issues facing teaching on the cloud from three perspectives: technology, organization and environment. It will use a theoretical framework called "TOE" which has three constructs each correspond to a factor influence the selection or changing of new technology in organizations.

The remainder of this paper is divided into the following sections:

- $\quad$ The paper first explains the theoretical framework used in this study 


\section{Issues in Information Systems}

Volume 20, Issue 4, pp. 147-156, 2019

- It then reviews literature regarding cloud computing, different definitions noted, and different terms used to give background information about the topic.

- A discussion is followed based on the literature review and the experience of the faculty teaching on the cloud the first time in a previous year.

- The conclusion for the framework and the factors in the framework is introduced at the end.

\section{THEORETICAL FRAMEWORK}

This study uses a theoretical framework called Technology, Organization and Environment (or TOE) to identify the aspects that influences the adoption of new technologies in organization. The TOE framework was first developed in 1990 by Depietro, Wiarda, and Fleischer, and it has been implemented since in various studies to identify the influence of different factors on introducing new technologies into an organization (Oliveira \& Martins, 2011). It identifies three constructs that influence such introduction of innovation: Technology, Organization and Environment. Figure 1 below depicts the relationship among these three constructs of the TOE framework:

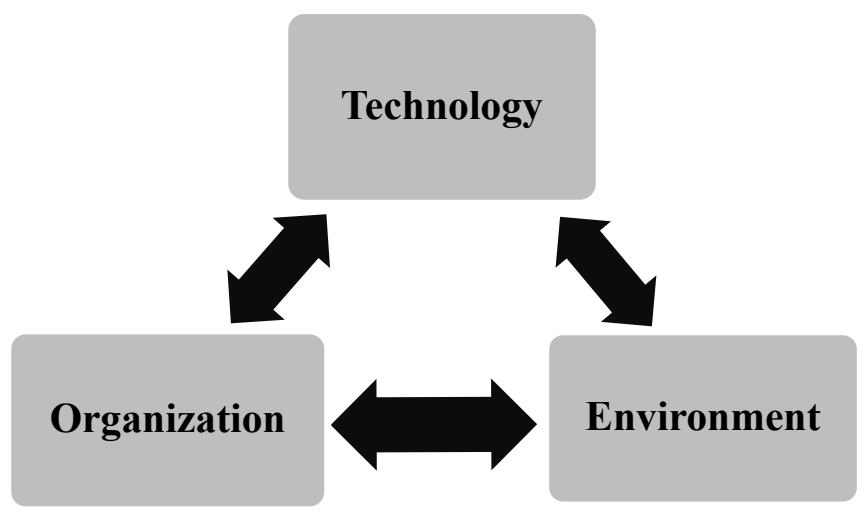

Figure 1: TOE Foundational Framework (extracted from Depietro, Wiarda, E. and Fleischer, 1990)

What is presented in figure 1 is the beginning foundation of the TOE framework. As the literature review is conducted, factors within each construct are identified and listed accordingly. On completion, the TOE framework yields a model conforming to the template shown in figure 2 . 


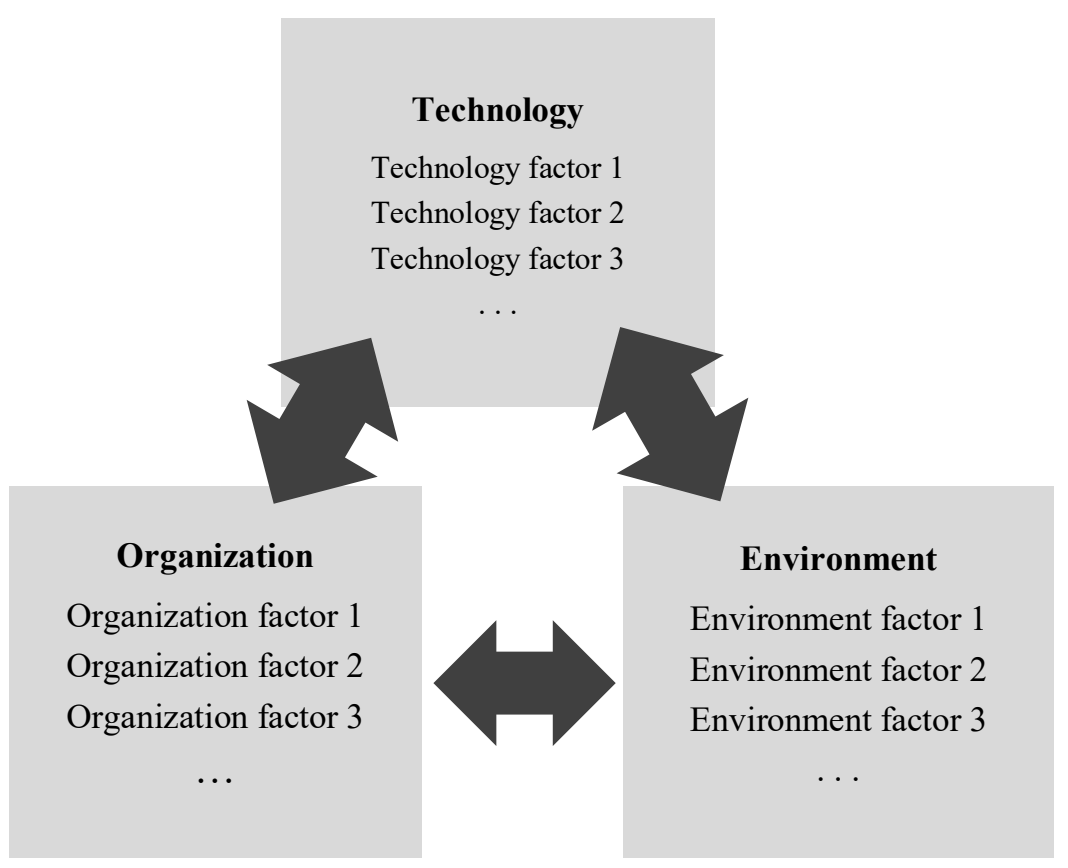

Figure 2: TOE Model Template

In this research, we are going to follow this process by including the factors that are needed to be taken into consideration under each construct as shown below:

- Technology construct: the technologies that are considered in this course of cloud computing when when teaching it on the cloud

- Organization construct: the organizational changes that are required in order to teach this course on the cloud

- Environment construct: the new working environmental factors that are considered when teaching on the cloud

This is consistent with the study made by Tashkandi and Al-Jabri (2015) and the study conducted by Low, Chen, and $\mathrm{Wu}(2011)$ when they attempted to understand the determinants of cloud adoption on organizations in general. However, our study focuses on the adoption of cloud computing when teaching on the cloud. By teaching on the cloud, we mean the course work (projects, exams and assignments) are completed on the cloud platform.

\section{LITEARATURE REVIEW - TECHNOLOGY OF CLOUD COMPUTING}

Although the term "cloud computing" is widely exchanged and repeated in the news, in textbooks and on the Internet, the exact meaning is not clear. This section gives background information about cloud computing, some definitions and the main characteristics. It differentiates between public and private cloud and introduces other background information.

\section{Cloud Computing Definitions}

There are many terms and definitions used and introduced to define and explain cloud computing. We have reviewed some definitions and selected four of them that explain cloud computing from diverse point of views. Table 1 below presents these definitions; the first column lists the reference while the second lists the definition. 
Table 1. Definitions of Cloud Computing

\begin{tabular}{|l|l|}
\hline Source & Definition \\
\hline $\begin{array}{l}\text { Mell \& Grance (2011), p. 2. } \\
\text { Based on the definition of the } \\
\begin{array}{l}\text { National Institute of Stand- } \\
\text { ards and Technology (NIST) }\end{array}\end{array}$ & $\begin{array}{l}\text { Cloud computing is a model for enabling ubiquitous, convenient, on-demand net- } \\
\text { work access to a shared pool of configurable computing resources (e.g., networks, } \\
\text { servers, storage, applications, and services) that can be rapidly provisioned and } \\
\text { released with minimal management effort or service provider interaction. }\end{array}$ \\
\hline Murah (2012),p. 157 & $\begin{array}{l}\text { Cloud computing is a technology that allow the users to access software applica- } \\
\text { tions, hardware, storage, computing processes directly from the web. It offers two } \\
\text { paradigms in computing; SaaS and PaaS. } \\
\text { Cloud computing technology is increasingly being used by enterprises and organ- } \\
\text { izations. It basically involves a variety of independent technologies such as hard- } \\
\text { ware virtualization, distributed processing, utility computing, network system, } \\
\text { web services, platform as a service, and software as a service. } \\
\text { Cloud computing is a form of computing where the IT needs of a consumer can be } \\
\text { bought from a cloud service provider. Another definition of cloud computing is a } \\
\text { computing model based on a customer service needs. }\end{array}$ \\
\hline Haris \& Khan (2018), p. 632 & $\begin{array}{l}\text { Cloud Computing is the fastest growing technology in the IT world. It is an archi- } \\
\text { tecture which combined the concept of Virtualization technology with several } \\
\text { computing paradigms such as Distributed computing, Utility computing, Grid } \\
\text { computing etc. to achieve the goal of providing unlimited resources and services } \\
\text { over the internet. }\end{array}$ \\
\hline Almajalid (2017), p. 2 & Pool of computing resources that are delivered on the web. \\
\hline
\end{tabular}

While there are many different definitions and approaches to explain cloud computing, the essential definition can be best expressed in terms of its main characteristics.

\section{Main Characteristics of Cloud Computing}

Mell and Grace (2011) summarized the characteristics that can distinguish cloud computing from traditional on premise computing:

- On demand self-service. This means that the services provided on the cloud are demanded (or consumed) by the others.

- Broad network access. Cloud computing places (or data centers) offer broad network access to services that enable their subscribers to connect to them through standard networks.

- Resource pooling. Resource services can be pooled between multiple organizations that can be purchased and be limited to those specified in the resource pooling.

- Rapid elasticity. Resource services available to a particular organization can range from simple to comprehensive, and can be re-provisioned with relative ease.

- Measured service. The utilization of a service is measured in some ways such as hours accessed, space provided, number of users allowed and so forth.

\section{Private and Public Cloud}

Private cloud are platforms created by different companies where the resources are only available for their own use. In other words, the cloud services for these companies are not available to the public, instead they are only available to individuals given access granted by this company (typically employees of the company that owns the cloud). Companies that provide private clouds for their computing services are on the rise. More and more companies are switching to the cloud to provide services for their own business.

Public cloud on the other hand is explained as computing services offered by third-party providers over the Internet, making them available to anyone who wants to use or purchase those services (Black, 2017). Public cloud services could be offered for free or sold on-demand, allowing customers to pay only per usage for applications, CPU cycles, 
storage, or bandwidth they consume (Moskalenko, 2017). Harvey (2017) listed four public cloud providers that are dominant in this field:

- $\quad$ Amazon Web Services

- $\quad$ Microsoft Azure

- $\quad$ IBM Cloud

- Google Cloud Platform

\section{Other Cloud Platforms}

This includes cloud platforms that are not limited to the four prominent listed above. Instead, rapid technological advances led to the introduction of other different platforms that provides services in data centers (or the cloud) and work similarly to what is available on the cloud platforms noted above. Alamajalid (2017) explained about two of these: Microsoft Live@edu and Google App for education (GAE) that enable better student collaboration. We visited the web site for the Google App for Education (https://edu.google.com) and found that they have a lot of teaching resources, some of which include: Activity, applied digital skills, Virtual Reality Creation Tool, Creativity tool, Google Artificial intelligence, Google arts and culture and many others.

Another platform that is considered when teaching on the cloud is Office 365 . When talking about office 365 , it is often thought of as extension to office 2016 and may understood that it is limited to the productivity software of Word, Excel, PowerPoint. Outlook, Publisher and Access. However, office 365 is a new technology that contains significantly more software. Barton (2015) listed the following software that can be included in MS Office 365:

- $\quad$ Microsoft OneDrive for business

- Microsoft Dynamics CRM Online

- $\quad$ Microsoft InTune

- Server manager

- Windows App Studio

We also visited the web site for office 365 (https:/www.office365.com) and realized that it contains a lot more software for educational purpose such as: Exchange, SharePoint, Skype, Sway, Forms, Flow, PowerApps, Booking, Power $\mathrm{BI}$ and Microsoft Team.

\section{Cloud Computing Concerns}

Moving computing resources to the cloud is not without problems. Instead, such a move is often faced with issues and concerns that arise as different organizations abandon their familiar on-premise computing to the less familiar computing on the cloud. Almajalid (2017) for example noted a few concerns about cloud that includes vendor lock, latency, reliability, security, control, performance, as well as privacy. Ganware and Ramaswamy (2015) noted the following risks and concerns from moving to the cloud: security, performance, higher cost, difficult integration with on-premise applications and difficulty customization process.

Kaufman (2009) noted data security in the world of cloud computing is a top concern. Kaufman further explained that the environment of cloud computing "require an implicit level of trust as well as an explicit level of vigilance to ensure success (p. 62)". Harris and Khan (2018) on the other hand explained about the payment method in cloud computing - which is the pay as per use basis. This payment requires paying as you go, if the organization use more computing resources, they pay more, while the opposite is correct.

The most relevant concerns to our study are security, privacy and the payment method (pay as you go). This is true as they organization moves on to a new and less familiar technology. This means that data, which is the most critical element that is typically hacked, is saved on external platforms without the safe guard procedures familiar to the organization.

The pay per use method is particularly problematic if teaching content on the cloud. It means that students must give a credit card number (or other payment method) and as they work on the platform and are charged according to usage. Ali and Smith (2018) noted that students are not comfortable with their method of payment, they are concerned about giving their credit card numbers to some mysterious institutions on the cloud.

The issue of pay-per-use does not end there. Instead, it has other issues with it. The case of pay per use means that if a student (A) works more on the platform than another student (B), then student A pays more for using the materials. 


\section{Issues in Information Systems \\ Volume 20, Issue 4, pp. 147-156, 2019}

This conflicts with purchasing textbooks and eBooks in other typical courses where students pay a fixed amount for textbooks and this does not change (and does not increase) per use. It potentially means the students pay the same price for the course materials. But in the case of teaching on the cloud, students may not pay the same for the course materials. One other issue that we did not find answer to with this pay-per-use method is the level of usage. If a student working on the cloud on and leaves it on for days, they be could be charged for it.

\section{Searching for Teaching Topics on the Cloud}

Our next step is to search and review literature to find topics on teaching in cloud computing. We first started to look at the foundation and the core concepts that cloud computing is based on. We found that virtualization is the key concept that is suggested in some literature as the foundation for cloud computing. Almajalid (2017) explained that what led to an increased use of cloud computing is that resources can be virtualized over the Internet. Haris and Khan (2018) noted that cloud computing is an advancement of various combined technologies such as distributed computing, utility computing, virtualization and others to provide IT services over the Internet on pay per use manner. Hassan, Mohamed and Sheta (2015) noted that through virtualization techniques multiple computing resources can be managed. The point to explain here is that virtualization is at the core of cloud computing and may need to be introduced in any given course in cloud computing.

In our search for teaching content on cloud computing we continue to review materials regarding Microsoft Azure (or Windows Azure). As noted before, Microsoft Azure is one of the leading public cloud computing platforms. It allows the use of different applications as a service, for different organizations. Chappell (2009) noted that that Windows Azure can be used as a foundation for running applications and storing data on the cloud. Chappell further gave an example to clarify what is meant that Azure is a service by saying "Rather than providing software that Microsoft customers can install and run themselves on their computers, Windows Azure today is a service: customers use it to run applications and store data on Internet-accessible machines owned by Microsoft (p. 2)".

We searched Windows Azure portal web site (www.portal.microsoft.com) to find some contents that can be suggested to include in our teaching materials. Figure 3 below shows a screen capture of what Azure Portal looks like when a user login in to it.

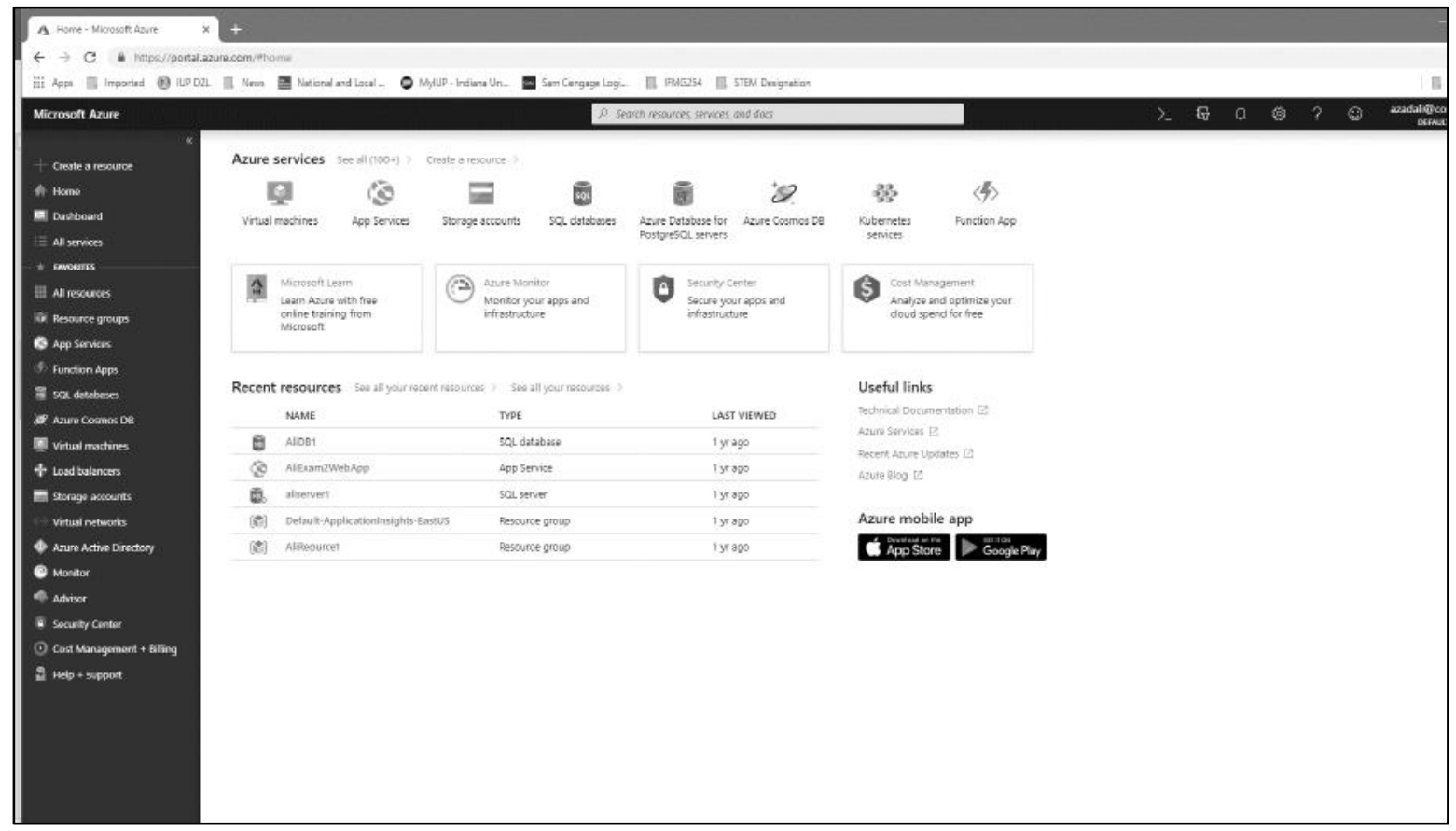

Figure 3. Windows Azure Portal

The portal has different applications listed horizontally and vertically. The horizontal line on top of the Azure portal screen has the following list of topics: 
- Virtual machine

- App services

- Storage accounts

- SQL database

- Azure Database for PosgreSQL servers

- Azure Cosmos DB

- Kubernetes services

- Functions apps

The applications list is not limited to the horizontal side, the vertical side has a list of applications that can be accessed as well. On the vertical side, Azure platform has the following additional links:

- Function apps

- Load Balancers

- $\quad$ Storage accounts

- Virtual network

- Security adviser

Each link can be a topic of teaching and discussion. When clicking on each link different help materials are available on the topics. This includes videos and tutorials an also links to additional resources.

As our literature search continued, we found different books published by Microsoft Press that suggested main topics in chapters of their books. Collier and Shahan (2015) for example suggested the following topics as the essentials and fundamentals of Microsoft Azure:

- Azure portal

- Azure web site and Azure cloud services

- Azure virtual machine

- Azure storage

- Azure virtual net works

- Azure databases

- $\quad$ Azure active directory

Tulloch (2013) suggested the following topics in for introducing Windows Azure for IT professionals:

- Windows Azure Computer services (web site, virtual machine, cloud services)

- Windows Azure Network Services (Virtual network, traffic manager)

- Windows Azure data services

- Data Management, HDInsight

- Business Analysis

- Windows Azure app services (Active directory, Multi-factor authentication, BizTalk services, Media services)

Guthrie et al. (2014) suggested the following main topics to study in Azure:

- Windows automation with DevOps

- Automation scrips

- Continuous integration and continuous delivery

- Web development best practices

- Data storage options and data partitioning strategies

\section{DISCUSSION}

There are a few factors that differ between teaching in a regular setting (we call it "on-premise computing") with teaching on the cloud. We will try here to explain the factors based on our experience teaching it the first time and 
based on the literature review presented earlier. We divide our discussion into three sections consistent with the three constructs of the TOE model that we introduced in this paper: Technology, Organization, Environment.

\section{Technology}

We discuss here what technologies we plan to teach based on the vast technologies available in within cloud computing platform. We selected two platforms to use when teaching on the cloud: first Microsoft Azure and second Microsoft Office 365 . We explain this later within our discussion of the organization construct.

First, we thought that it is imperative to teach Virtualization within the Azure framework. Virtualization is the basis at which cloud computing stands and it is important to for students to gain experience with it. We suggest having students create a virtual machine with which students can use applets, assign users, give permissions and perform other administrative activities.

Among the services listed in the Azure platform, we thought it will be necessary to introduce some applications here. Among what we think helpful are the following apps:

- App services

- $\quad$ Storage accounts

- SQL database

- Functions apps

- Virtual network

From the office 365 we have determined that it will be helpful to introduce our students to the following software on the cloud:

- $\quad$ Microsoft OneDrive

- Microsoft Sway

- Microsoft Flow

- $\quad$ Microsoft form

\section{Organization}

The organizational aspect we went through is the Azure platform and the Office 365. We decided to go with Azure to take advantage of the Microsoft Imagine Academy (https://member.imagineacademy.microsoft.com) agreement. Through this academy, students have free access to some software and applications. Specifically, through the academy they will be able to get access the following app services without additional cost: App services, Storage accounts, SQL database and Functions apps. For the Microsoft office, our institution is included in the Microsoft virtual academy agreement by which our students receive free access to office 365 .

\section{Environment}

The environment in which we are to work with this different software proved to be tricky. The pay-as-go proves to be difficult to manage. Students are confronted with a new platform where they have to give credit card number to get access to these services. We tried finding a different arrangement where the students can pay in advance for the services but did not find this practice available with Microsoft for their Azure product.

\section{CONCLUSION}

The objective that we stated at the beginning of this paper is to discuss the platform of teaching cloud computing within the constructs of the TOE framework. As noted before, the TOE framework has three constructs and each construct has their own element. After going though our experience teaching cloud computing the first time and after doing the literature review here, we decided on the elements that goes under each construct of the TOE model. The suggested application of the TOE model for teaching on the cloud for our course is presented in the figure 4 below. 


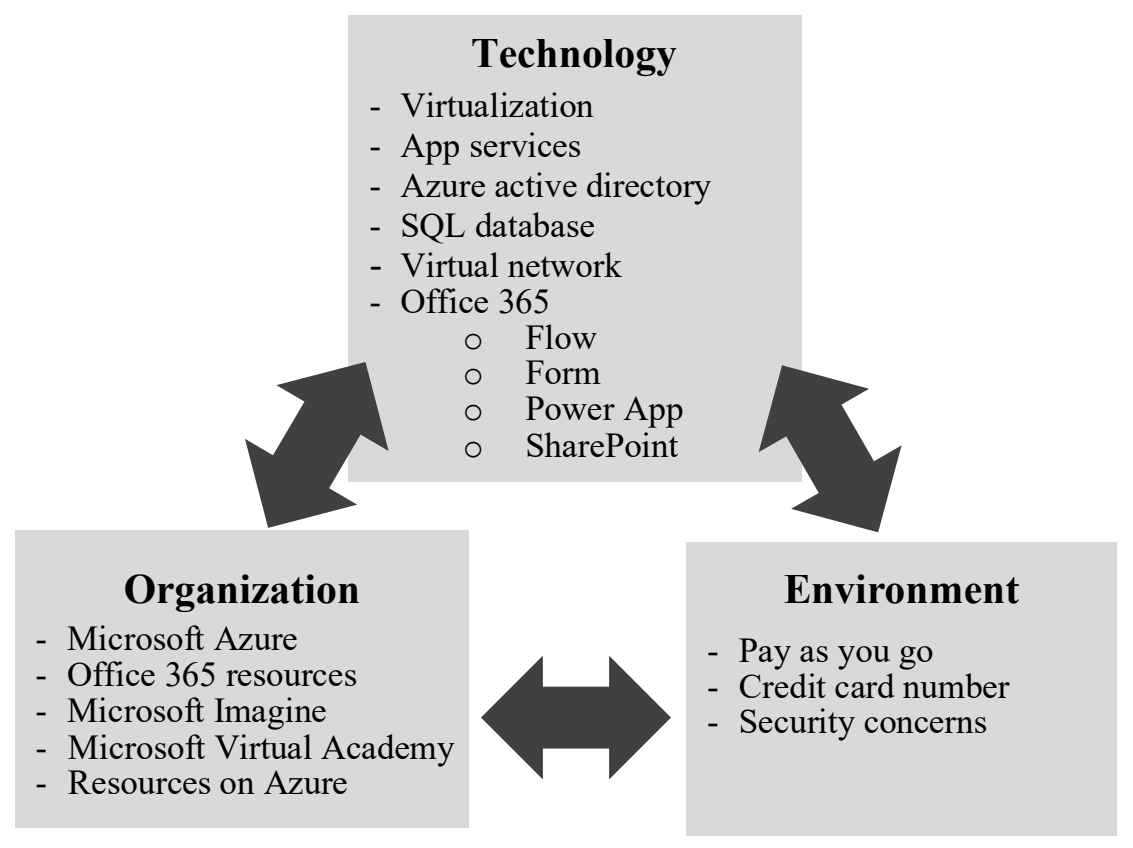

Figure 4. Resulting TOE Model for Teaching on the Cloud

\section{REFERENCES}

Alharthi, A., Alassafi, M. O., Walters, R. J., \& Wills, G. B. (2017). An exploratory study for investigating the critical success factors for cloud migration in the Saudi Arabian higher education context. Telematics and Informatics, 34(2), 664-678.

Ali, A., Smith, D. T., \& Leslie, T. A. (2018). Issues and Challenges Facing The Teaching of Cloud Computing For The First Time. Issues in Information Systems, 19(4), 187-195.

Almajalid, R. (2017). A survey on the adoption of cloud computing in education sector. arXiv preprint arXiv:1706.01136.

Barton, B. (2015). Microsoft Public Cloud Services: Setting up your business in the cloud. Microsoft Press.

Chappell, D. (2009). Introducing windows Azure, Microsoft, Dec.

Collier, M., \& Shahan, R. (2015). Microsoft Azure Essentials-Fundamentals of Azure. Microsoft Press.

DePietro, R., Wiarda, E. \& Fleischer, M. (1990). The context for change: organization, technology and environment, In Tornatzky, L.G. \& Fleischer, M. (Eds), The Process of Technological Innovation, (pp. 151-175). Lexington Books, Lexington, MA,

Gangwar, H., Date, H., \& Ramaswamy, R. (2015). Understanding determinants of cloud computing adoption using an integrated TAM-TOE model. Journal of Enterprise Information Management, 28(1), 107-130.

Guthrie, S., Simms, M., \& Dykstra, T. (2014). Building Cloud Apps with Microsoft Azure: Best practices for DevOps, data storage, high availability, and more. Pearson Education.

Hassan, H. A., Mohamed, S. A., \& Sheta, W. M. (2016). Scalability and communication performance of HPC on Azure Cloud. Egyptian Informatics Journal, 17(2), 175-182. 


\section{Issues in Information Systems}

Volume 20, Issue 4, pp. 147-156, 2019

Haris, M., \& Khan, R. Z. (2018). A Systematic Review on Cloud Computing. International Journal of Computer Sciences and Engineering, 6(11), 632-639.

Harvey, C. (2017). Public Cloud Computing Providers. Datamation. Retrieved from https://www.datamation.com/cloud-computing/public-cloud-providers.html.

Kaufman, L. M. (2009). Data security in the world of cloud computing. IEEE Security \& Privacy, 7(4), 61-64.

Low, C., Chen, Y., \& Wu, M. (2011). Understanding the determinants of cloud computing adoption. Industrial management \& data systems, 111(7), 1006-1023.

Moskalenko, A. (2017). Deciding If the Cloud Is Right for Your Law Practice. Gpsolo, 34(4), 60-63.

Murah, M. Z. (2012). Teaching and learning cloud computing. Procedia-Social and Behavioral Sciences, 59, 157163.

Oliveira, T., \& Martins, M. F. (2011). Literature review of information technology adoption models at firm level. Electronic Journal of Information Systems Evaluation, 14(1), 110.

Tulloch, M. (2013). Introducing Windows Azure for IT Professionals. Microsoft Press.

Tashkandi, A. N., \& Al-Jabri, I. M. (2015). Cloud computing adoption by higher education institutions in Saudi Arabia: an exploratory study. Cluster Computing, 18(4), 1527-1537.

Tornatzky, L. and Fleischer, M. (1990) The process of technology innovation, Lexington, MA, Lexington Books. 